\title{
ARTICLE \\ Adipose-derived mesenchymal stem cells protect against CMS-induced depression-like behaviors in mice via regulating the Nrf2/HO-1 and TLR4/NF-kB signaling pathways
}

\author{
Xiao Huang ${ }^{1,2}$, Guo-qiang Fei ${ }^{2}$, Wen-juan $\mathrm{Liu}^{1}$, Jing Ding ${ }^{2}$, Yuan Wang ${ }^{1}$, Hao Wang ${ }^{3}$, Jian-lin Ji ${ }^{1}$ and Xin Wang ${ }^{2,4}$
}

\begin{abstract}
Increasing studies show that inflammatory processes may be involved in depressive disorders. Nuclear factor erythroid-2 related factor 2 (Nrf2) modulates tissue microglial M1 phenotypic changes to the M2 phenotype, which is implicated in protection against inflammatory diseases. We have reported that the adipose-derived mesenchymal stem cells (ADSCs) display anti-inflammatory activity. In this study we explored whether the mechanism of anti-inflammatory activity of ADSCs was related to Nrf2. ADSCs were isolated from mouse fat pads and intravenously administered to chronic mild stress (CMS)-exposed C57BL/6 mice at the dose of $1 \times$ $10^{6}$ once a week for 3 weeks. We showed that ADSC administration significantly remedied CMS-induced depressive-like behaviors in sucrose preference test, tail suspension test, and forced swim test accompanied by suppressing microglial activation and the expression of inflammatory factors including MCP-1, TNF- $\alpha, I L-1 \beta$, and IL- 6 . Furthermore, ADSC administration promoted both the expression of BDNF and TrkB, and promoted Nrf2/HO-1 signaling but suppressed TLR4/NF-KB signaling in brain tissue. In order to elucidate the role of Nrf2/HO-1 signaling in ADSC-caused neuroprotection, Nrf2-modified ADSCs were cocultured with BV2 microglial cells, then exposed to lipopolysaccharide (LPS). Downregulation of Nrf2 in ADSCs decreased the protective effects of ADSCs against LPS-induced microglial activation and M1 polarization. Nrf2 overexpression in ADSCs markedly suppressed LPSinduced TLR4 and NF-KB expression in microglial cells. These results suggest a possible antidepressive mechanism correlated with microglial polarization for anti-inflammatory agents, which may provide a new microglia-targeted strategy for depression therapy.
\end{abstract}

Keywords: ADSCs; Nrf2; TLR4; microglial; chronic mild stress

Acta Pharmacologica Sinica (2020) 41:612-619; https://doi.org/10.1038/s41401-019-0317-6

\section{INTRODUCTION}

Depression, a common multicausal psychiatric disorder and a significant contributor to the social burden of disease, is characterized by a lack of interest, sleep disturbances, poor concentration, enduring sadness, and suicidal tendencies [1]. Increasing evidence suggests that inflammation and immune activation may be closely related to the pathogenesis of depression [2-5]. Clinical findings have indicated that only $30 \%$ of depressive patients achieve full remission (Hamilton rating score $\leq 7$ ) [6]. Therefore, developing new targets based on the understanding of the pathophysiological mechanisms associated with depression is urgently required for the study of novel antidepressants.

Microglia are resident innate immune cells within the central nervous system (CNS) and play a central role in the neuroinflammatory response $[7,8]$. In clinical research, microglial activation has also been observed in patients with depression who have committed suicide [9]. Activated microglia are often categorized into classical (M1) and alternative (M2) phenotypes. M1 microglia may contribute to the dysfunction of the neurotrophic system by expressing proinflammatory cytokines, such as tumor necrosis factor- $a$ (TNF- $\alpha$ ), IL-1 $\beta$, IL-6, iNOS, and CCL2, which lead to neurotoxic outcomes [10]. The M2 phenotype, sometimes called the neuroprotective microglial phenotype, induces the release of different mediators, including Ym1, arginase 1 (Arg1), IL-10, IL-4, and TGF- $\beta$, to antagonize inflammation-induced damage in the CNS [11, 12]. These data indicate that the M2 microglial phenotype may have a therapeutic effect on depression. Interestingly, previous studies have found that nuclear factor-E2 related factor 2 (Nrf2) has an important suppressive effect on the inflammatory response by inhibiting the nuclear factor-KB (NF-KB) signaling pathway $[13,14]$. Previous studies have found that the activation of TLR4 signaling promotes the phosphorylation of NF-KB, thereby promoting inflammatory effects [15]. The expression of the TLR4/NF-KB signaling pathway was found to be negatively correlated with the expression of Nif2 [16]. The increased activation of Nrf2 along with its target antioxidant enzyme HO-1 was also shown to inhibit the expression of TLR4/MyD88 [17]. Finally, a study found that Nrf2 activation reduces brain inflammation after stroke by enhancing microglial M2 polarization [18].

Adipose-derived mesenchymal stem cells (ADSCs) are easily isolated and expanded from adipose tissues. ADSCs are multipotent cells that can be induced to differentiate into many lineages, including adipogenic, chondrogenic, myogenic, and

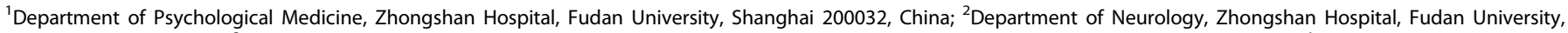

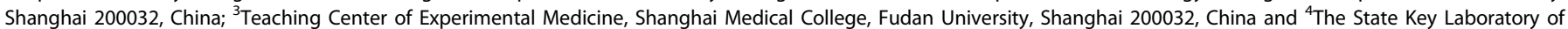
Medical Neurobiology, the Institutes of Brain Science and the Collaborative Innovation Center for Brain Science, Fudan University, Shanghai 200032, China Correspondence: Hao Wang (wh91351@126.com) or Jian-lin Ji (ji.jianlin@zs-hospital.sh.cn) or Xin Wang (wang.xin@zs-hospital.sh.cn)

Received: 31 May 2019 Accepted: 9 October 2019

Published online: 3 December 2019 
osteogenic cells [19]. After transplantation, ADSCs can engraft in brain tissue and differentiate in situ into neurons and glial cells, and as a result, ADSCs are usually used in peripheral nerve regeneration [20-22]. Recently, studies have found that ADSC transplantation therapy has anti-inflammatory effects [23, 24]. In the present study, we studied the therapeutic mechanism of ADSCs in depression and sought to elucidate the relationship between Nrf2 and microglial phenotype by using a chronic mild stress (CMS)-induced depressive mouse model and lipopolysaccharide (LPS)-induced inflammatory cell model.

\section{MATERIALS AND METHODS}

Male C57BL/6 mice (7-8 weeks old) were purchased from SLAC Laboratory Animal Co. Ltd. (Shanghai, China). All animal experiments were approved and performed according to the guidelines of the Ethics Committee of Zhongshan Hospital of Fudan University, Shanghai, China. All surgical procedures were performed under anesthesia, and every effort was made to minimize suffering.

Isolation, culture, and identification of ADSCs

ADSCs were isolated as previously described [24]. Briefly, adipose tissue from mouse abdominal fat pads was isolated and washed with phosphate-buffered saline (PBS) and minced before digestion with $0.2 \%$ collagenase I (Sigma-Aldrich, St. Louis, MO, USA) for $1 \mathrm{~h}$ at $37^{\circ} \mathrm{C}$ with intermittent shaking. The digested tissue was washed with Dulbecco's modified Eagle's medium (DMEM, Sigma-Aldrich) containing $15 \%$ fetal bovine serum (FBS, Gibco BRL, MD, USA) and centrifuged at $1200 \times g$ for $10 \mathrm{~min}$ to remove mature adipocytes. The pellet was resuspended in DMEM supplemented with $15 \%$ FBS, $100 \mathrm{U} / \mathrm{mL}$ penicillin, and $100 \mu \mathrm{g} / \mathrm{mL}$ streptomycin and cultured at $37{ }^{\circ} \mathrm{C}$ and $5 \% \mathrm{CO}_{2}$. ADSCs that reached $80 \%-90 \%$ confluency were detached with $0.02 \%$ ethylenediaminetetraacetic acid/0.25\% trypsin (Sigma-Aldrich) for $5 \mathrm{~min}$ at room temperature and then replated. For phenotypic analysis, fluorescein isothiocyanate- or phycoerythrin-conjugated CD29, CD90, CD44, CD105, and VWF antibodies were used. An IgG-matched isotype served as the internal control for each antibody. Normoxic ADSCs were cultured in $95 \%$ air $\left(20 \% \mathrm{O}_{2}\right)$ and $5 \% \mathrm{CO}_{2}$.

Multilineage differentiation of ADSCs

To evaluate the multilineage differentiation of ADSCs, third-passage mouse ADSCs were cultured in adipogenic differentiation medium and stained with oil red $\mathrm{O}$ after 14 days or cultured in osteogenic differentiation medium and stained with alizarin red after 21 days.

\section{RNA interference or overexpression}

To modify Nrf2 expression in ADSCs, an Nrf2 interference vector (siNrf2) and an Nrf2 overexpression vector were synthesized (GenePharma, Shanghai, China). Then, ADSCs were transferred to six-well culture plates and transfected by incubation in complete medium containing $100 \mu \mathrm{g} / \mathrm{mL}$ ADSC-exosomes (200 $\mu \mathrm{g} /$ well) and Lipofectamine 2000 (Thermo Scientific, MMAS, USA) or an equal volume of PBS for $48 \mathrm{~h}$ before exosomes were isolated for other experiments.

Microglial cell culture and treatment

BV2 microglial cells (FuHeng Biology, Shanghai, China) were cultured in DMEM (Sigma-Aldrich) with 10\% FBS (Sigma-Aldrich), $100 \mathrm{U} / \mathrm{mL}$ penicillin (Invitrogen, CA, USA), and $0.1 \mathrm{mg} / \mathrm{mL}$ streptomycin (Invitrogen, CA, USA) in a humidified atmosphere of $5 \% \mathrm{CO}_{2}$ and $95 \%$ air at $37^{\circ} \mathrm{C}$. The cells were passaged two to three times per week and then distributed into 24-well plates at a density of $5 \times 10^{5}$ cells per well. The cultivated BV2 cells were treated with or without $100 \mathrm{ng} / \mathrm{mL}$ LPS (Sigma-Aldrich) or with $100 \mu \mathrm{M}$ Exo $(100 \mu \mathrm{g} / \mathrm{mL})$. After culturing for $24 \mathrm{~h}$, the cells were analyzed using real-time polymerase chain reaction (RT-PCR), enzymelinked immunoabsorbent assay (ELISA), and Western blotting.

\begin{tabular}{|ll|}
\hline Table. 1. & The primer sequences \\
\hline Gene name & Primer $\left(5^{\prime}\right.$ to $\left.3^{\prime}\right)$ \\
\hline$\beta$-actin & Sense: CCGTGAAAAGATGACCCAGATC \\
iNOS & Antisense: CACAGCCTGGATGGCTACGT \\
& Sense: GCAGAGATTGGAGGCCTTGTG \\
Arg1 & Antisense: GGGTTGTTGCTGAACTTCCAGTC \\
& Sense: GGAAGACAGCAGAGGAGGTG \\
Ym1 & Antisense: TATGGTTACCCTCCCGTTGA \\
& Sense: TCACTTACACACATGAGCAAGAC \\
CCL2 & Antisense: CGGTTCTGAGGAGTAGAGACCA \\
& Sense: CTGATGCAGGTCCCTATGGT \\
& Antisense: GCAGGATTTTGAGGTCCAGA
\end{tabular}

RNA extraction and RT-PCR

Total RNA was isolated from brain tissues or cells using TRlzol reagent. First-strand cDNA was synthesized using the PrimeScript $^{\text {TM }}$ RT Master Mix (Perfect Real Time) Kit (Takara Bio Inc., Shiga, Japan), which, along with forward and reverse primers and Power SYBR Green PCR Master Mix (Life Technologies, Thermo Fisher Scientific, CA, USA), was then used for RT-PCR. $\beta$-Actin was used as the internal control. The primer sequences were shown in Table 1. The quantification of relative protein and endogenous control mRNA levels was performed using TaqMan assays. The data were analyzed using the $2^{-\Delta \Delta C t}$ method.

\section{ELISA}

BV2 cells or serum were treated with RIPA Lysis Buffer (Beyotime Institute of Biotechnology, China) and centrifuged at $12000 \times g$ for $5 \mathrm{~min}$. The supernatants were collected to detect the protein levels by ELISA. The expression of MCP-1, IL- 6 , TNF- $\alpha$, and IL-1 $\beta$ was assayed using ELISA kits (NeoBioscience Technology Co., Ltd., Beijing, China) according to the manufacturer's protocol. The absorbance at $450 \mathrm{~nm}$ was recorded using a microplate reader.

\section{Western blot analysis}

Total protein from brain tissue or BV2 cells was collected, and protein concentrations were determined with a BCA kit (Beyotime Institute of Biotechnology, China) following the manufacturer's guidelines. Equal amounts of protein were separated by SDSpolyacrylamide gel electrophoresis, transferred to polyvinylidene difluoride membranes (Millipore, MA, USA), blocked with 5\% skim milk, and incubated with primary antibodies (see below) either overnight at $4{ }^{\circ} \mathrm{C}$ or for $1 \mathrm{~h}$ at room temperature. The membranes were washed and incubated with a horseradish peroxidaseconjugated anti-rabbit IgG secondary antibody (1:500; Beyotime Institute of Biotechnology, China) and were visualized using an ECL Plus kit (Millipore). Densitometry was performed to quantify the signal intensity using ImageJ software (Version $1.45 \mathrm{~J}$; National Institutes of Health, Bethesda, MD, USA). The primary antibodies were rabbit anti-Nrf2, anti-HO-1, anti-NF-KB1, anti-CD29, antiCD90, anti-CD44, anti-CD105, anti-CD34, anti-vWF, anti-BDNF, antiTrkB, and anti-GAPDH (Abcam, Cambridge, UK).

\section{CMS procedure}

The CMS procedure was performed according to previously described $[25,26]$. The stress procedure followed a random schedule of commonly used mild stressors, such as food deprivation for $24 \mathrm{~h}$, water deprivation for $24 \mathrm{~h}$, cage tilting $\left(45^{\circ}\right)$ for $24 \mathrm{~h}$, damp bedding for $24 \mathrm{~h}$, stroboscopic illumination for $24 \mathrm{~h}$, overnight illumination and restraint stress for $2 \mathrm{~h}$. The mice received one stressor once per day, and the same stressor was never applied on 2 consecutive days. The stress regimen lasted for 6 consecutive weeks. The control group was housed in a separate 
room and had no contact with the stressed animals. After 3 weeks of the CMS procedure, the stressed mice were divided into the vehicle group and the drug-treated group. The drug-treated mice received an intravenous injection of ADSCs $\left(1 \times 10^{6}\right)$ once a week for 3 weeks. Body weight (BW) was measured every week. After 6 weeks of treatment, the mice in the different groups were used for behavioral measurements and pathological analysis.

\section{Behavioral measurements}

Sucrose preference test (SPT). Mice were subjected to the SPT weekly, as described previously $[25,26]$. Briefly, mice were housed individually and deprived of food for $6 \mathrm{~h}$ and water for $12 \mathrm{~h}$ before the test and then given access to water $(\mathrm{A})$ and $1 \%$ sucrose solution (B) for $2 \mathrm{~h}$ (both in bottles). The position of the two bottles was changed every $6 \mathrm{~h}$ to prevent possible side preference in drinking behavior. Then, the mice were deprived of food and water for $24 \mathrm{~h}$. On the testing day after 6 weeks of treatment, each mouse was exposed to preweighed bottles containing water and $1 \%$ sucrose solution for $1 \mathrm{~h}$ with their positions interchanged. Sucrose preference was calculated as a percentage of sucrose solution consumed relative to the total amount of liquid intake.

Tail suspension test (TST) and forced swimming test (FST). The TST and FST were performed according to previous studies [26, 27]. For the TST, the mice in the different groups were individually suspended $50 \mathrm{~cm}$ above the floor for $6 \mathrm{~min}$ by adhesive tape placed $\sim 1 \mathrm{~cm}$ from the tip of the tail. An investigator blinded to the study then recorded the duration of immobility during the last 4 min of suspension. The mice were considered immobile only when they hung passively and were completely motionless. Any mouse that climbed up its tail was excluded from further analysis. For the FST, the mice in the different groups were individually placed in a clear glass cylinder $(25 \mathrm{~cm}$ in height and $10 \mathrm{~cm}$ in diameter) filled to a height of $10 \mathrm{~cm}$ with water at $25 \pm 1{ }^{\circ} \mathrm{C}$ for 6 min. An investigator blinded to the study then recorded the duration of immobility during the animal's last $4 \mathrm{~min}$ in the water. The immobility time was defined as the time spent by the mouse floating in the water without struggling and making only those movements necessary to keep its head above the water.

Immunofluorescence. The animals were anesthetized, and the brain tissues were fixed with $4 \%$ paraformaldehyde. The tissues were cut coronally into $4-\mu \mathrm{m}$ slices with a sliding vibratome (CM1900; Leica Microsystems, Wetzlar, Germany). The immunofluorescence and statistical methods were based on previously described methods [28]. Brains sections that contained the hippocampus were permeabilized with $0.5 \%$ Triton X-100 for $10 \mathrm{~min}$. The samples were placed in $10 \%$ donkey serum for $2 \mathrm{~h}$ and incubated with a goat antiionized calcium-binding adapter protein-1 (lba1) antibody (1:600; Abcam) overnight at $4{ }^{\circ} \mathrm{C}$ and then incubated with a fluorescent dye-conjugated secondary antibody. The fluorescence was assessed under a fluorescence microscope.

Immunohistochemical assays. Apoptosis was assayed in fixed coronal sections with terminal deoxynucleotidyl transferasemediated dUTP-biotin nick end labeling using a commercially available in situ cell death detection kit (Roche Diagnostics).

\section{Statistical analysis}

Continuous variables are expressed as the mean \pm SEM. One-way ANOVA was performed for multiple comparisons using GraphPad Prism software, version 5.0 (GraphPad, La Jolla, CA, USA). P-values $\leq$ 0.05 indicate a statistically significant difference.

\section{RESULTS}

Characterization of ADSCs

Accumulating evidence has brought stem cell therapy to the forefront as a new promising approach for stroke treatment [29]. Furthermore, a study also found that stem cells, especially ADSCs, are a promising tool for neural regeneration and thus have become increasingly utilized in research [30]. Therefore, to verify whether ADSC therapy can improve cognitive dysfunction and hippocampal nerve damage associated with depression, we first isolated ADSCs from mouse fat pads (Fig. 1a). Immunofluorescence showed positive a

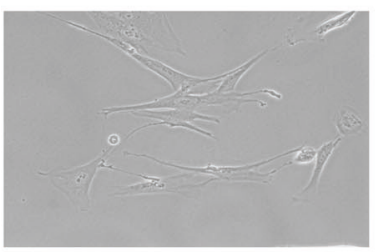

d

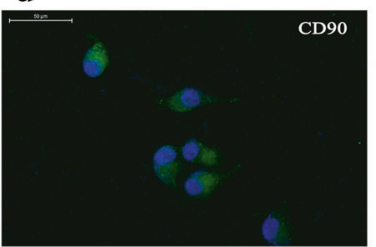

g

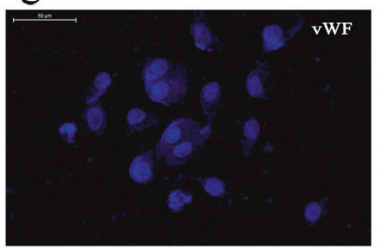

b

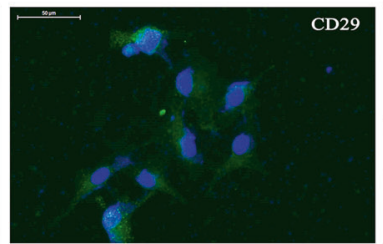

e

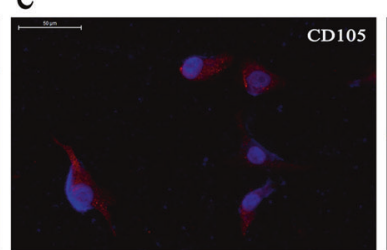

$\mathrm{h}$

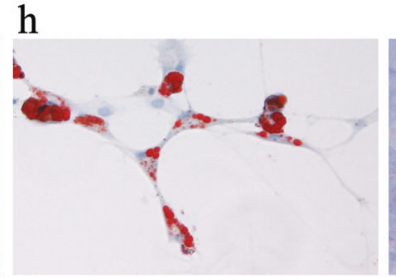

c

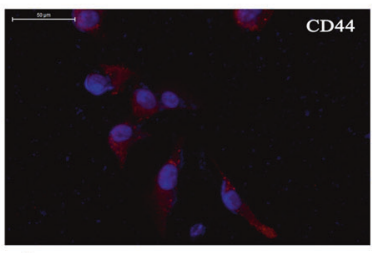

f

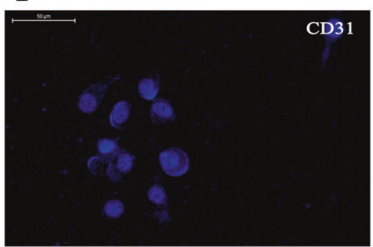

i

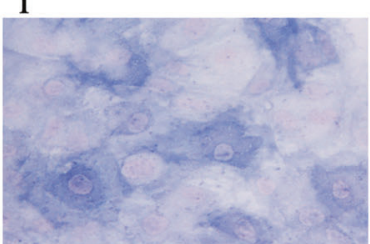

Fig. 1 Characterization of adipose-derived mesenchymal stem cells (ADSCs). a ADSCs showed a typical cobblestone-like morphology. Scale bar: $30 \mu \mathrm{m}$. b-g Immunofluorescence staining of CD29, CD90, CD44, CD105, CD34, and von Willebrand Factor (vWF). FITC- and PE-labeled mouse lgG isotype controls are shown $(\times 200)$. Scale bar: $50 \mu \mathrm{m}$. The differentiation potential of ADSCs assessed by oil red O (h) and alkaline phosphatase staining (i). Scale bar: $50 \mu \mathrm{m}$. 
staining for surface mesenchymal cell markers including CD29, CD90, CD44, and CD105 but negative staining for the endothelial markers CD34 and vWF (Fig. 1b-g). Finally, the results of oil red $\mathrm{O}$ and alizarin red staining confirmed adipocyte differentiation and osteoblast differentiation (Fig. 1h, i).

ADSC treatment reversed CMS-induced depressive-like behaviors The CMS experimental design is presented in Fig. 2a. Eighteen mice (control, CMS, and CMS + ADSCs) were used. The BW of the mice in the CMS group slowly decreased compared with that in the control group, and ADSC treatment restored the CMS-induced BW reduction (Fig. 2b, c). CMS-induced obvious depressive-like behaviors in mice, including a decrease in sucrose preference (Fig. 2d) and an increase in immobility in the TST (Fig. 2e) and FST (Fig. 2f). ADSC treatment significantly reversed CMS-induced depressive-like behaviors, suggesting that ADSC treatment improved CMS-induced depressive-like behaviors

ADSC treatment reversed CMS-induced inflammatory factor expression and hippocampal microglial polarization

Increasing evidence has revealed that inflammation in the context of the nervous system, termed neuroinflammation, is reported in patients with neuropsychiatric disorders and is typically associated

a

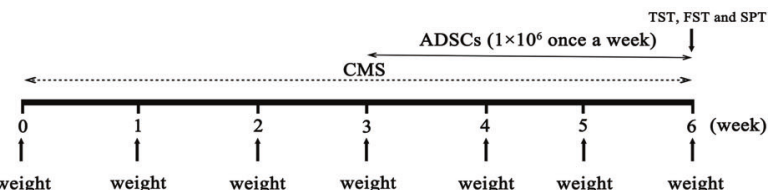

b
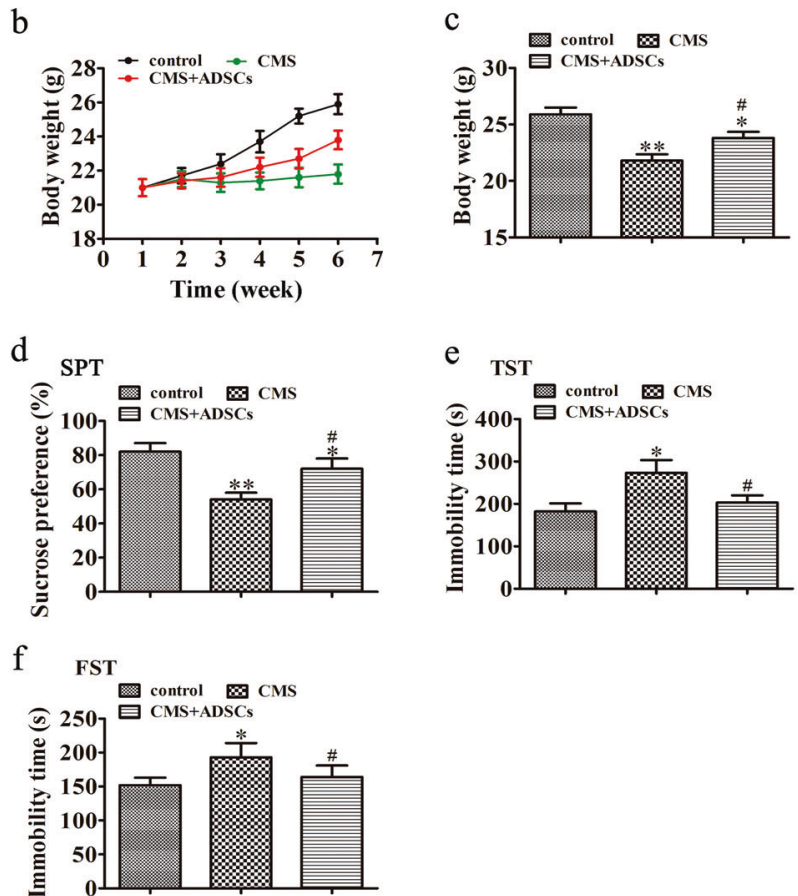

Fig. 2 ADSC treatment reversed chronic mild stress (CMS)induced depressive-like behaviors. a A schematic diagram of the experimental design. b Body weight (BW) was measured every week. c ADSC treatment $\left(1 \times 10^{6} /\right.$ week) induced the highest rate of BW recovery. The data are expressed as the mean \pm SEM $(n=10)$. ${ }^{*} P$ $<0.05,{ }^{*} P<0.01$ vs control, ${ }^{\#} P<0.05$ vs CMS. The regulatory effect of ADSCs on performance in the sucrose preference test (SPT) (d), tail suspension test (TST) (e), and forced swimming test (FST) (f) after CMS. The data are expressed as the mean $\pm \mathrm{SEM} ; n=10$. ${ }^{*} P<0.05$, ${ }^{*} P<0.01$ vs control; ${ }^{*} P<0.05$ vs CMS. with microglial activation and the production of cytokines [31-33]. In this study, we found that CMS significantly promoted MCP-1, TNF- $\alpha$, IL-1 $\beta$, and IL- 6 expression in the serum but that ADSC treatment reversed this CMS-induced inflammatory factor production (Fig. 3a-d). Immunofluorescence detection revealed that, 6 weeks after CMS induction, the number of $\mathrm{Iba} 1^{+}$microglia was increased compared with that in the control group but that the number of $\mathrm{Iba}^{+}$microglia decreased with ADSC treatment (Fig. 3e, f). Immunohistochemical detection also showed that, 6 weeks after CMS induction, the number of apoptotic neuronal
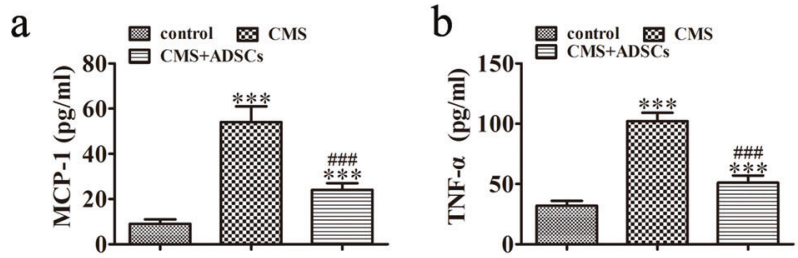

C

d
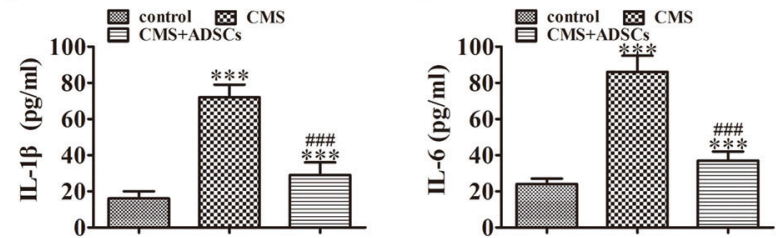

e
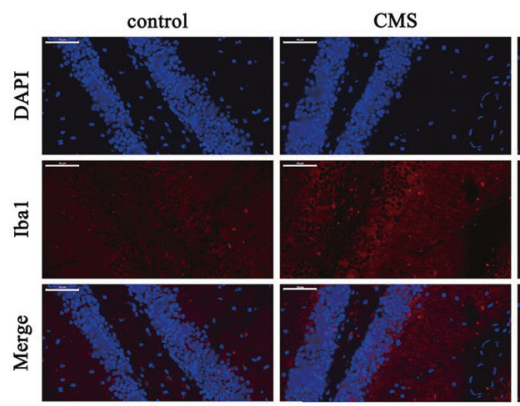

CMS+ADSCs

f

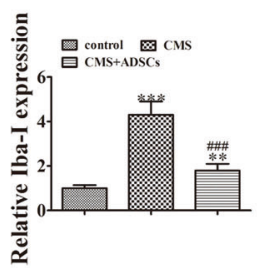

g

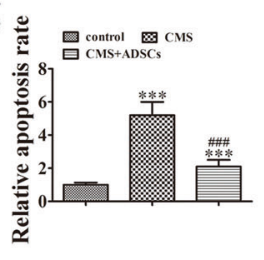

$\mathrm{h}$

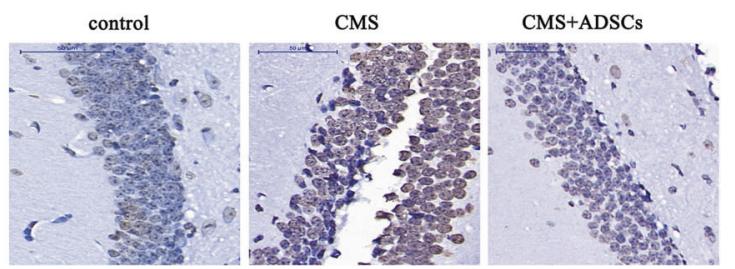

Fig. 3 ADSC treatment reversed CMS-induced inflammatory factor expression and hippocampal microglial activation. a-d ELISA analysis showing the expression of the inflammatory factors MCP-1, TNF- $\alpha$, IL-1 $\beta$, IL- 6 in the serum. The data are expressed as the mean \pm SEM $(n=10)$. ${ }^{* * *} P<0.001$ vs control. ${ }^{\# \#} P<0.001$ vs CMS. e, $\mathbf{f}$ Representative images showing that ADSC treatment decreased the number of $\mathrm{Iba}^{+}$microglia compared with that in the CMS mice. The data are expressed as the mean \pm SEM $(n=6) .{ }^{* *} P<0.01$, ${ }^{* * *} P<0.001$ vs control; ${ }^{* \# \#} P<0.001$ vs CMS. g, h Immunohistochemical detection of apoptotic hippocampal neurons. The data are expressed as the mean \pm SEM $(n=10)$. ${ }^{* *} P<0.001$ vs control; ${ }^{\# \#} P<$ 0.001 vs CMS. Scale bar: $50 \mu \mathrm{m}$. 
cells was increased compared with that in the control group but was decreased with ADSC treatment (Fig. 3g, h). These data showed that ADSCs exerted an inhibitory effect on CMS-induced microglial activation.

Western blot detection further showed that brain-derived neurotrophic factor (BDNF) and tyrosine receptor kinase B (TrkB) expression was decreased with CMS induction. In contrast, ADSC treatment promoted the expression of both BDNF and TrkB in brain tissue (Fig. $4 a-c)$. Previously, it was shown that BDNF-TrkB signaling is involved in the histopathological damage of hippocampal neuronal cells $[34,35]$ and suggested that TrkB and BDNF may play neuroprotective roles. This evidence reflected the protective effect of ADSCs in CMS-induced hippocampal neuronal cell damage.

We also found that Nrf2/HO-1 and TLR4/NFKB signaling were involved in CMS-induced depression in mice. CMS induction promoted TLR4/NFKB signaling but suppressed Nrf2/ HO-1 signaling, while ADSC treatment had the opposite effect (Fig. 4d-h).

Nrf2 played a role in the ADSC-mediated protective effects against LPS-induced inflammatory factor expression in BV2 microglial cells To confirm whether Nrf2/HO-1 plays a role in ADSC-mediated neuroprotection, we constructed an Nrf2 overexpression vector and siRNA targeting Nrf2 (siNrf2). The results showed that Nrf2 and HO-1 expression was increased in ADSCs after transfection with the Nrf2 overexpression vector but decreased after Nrf2 silencing (Fig. 5). ADSC and BV2 cell cocultures showed that the increased expression of TLR4 and NFKB induced by LPS was decreased by treatment with Nrf2-overexpressing ADSCs, but the downregulation of Nrf2 decreased the inhibitory effect of ADSCs on LPS-induced TLR4 and NFKB expression (Fig. 6a-c). ELISA showed that the upregulation of Nrf2 in ADSCs suppressed the secretion of the LPS-induced inflammatory factors MCP-1, TNF-a, IL-1 $\beta$, and IL- 6 by BV2 cells (Fig. $6 \mathrm{~d}-\mathrm{g}$ ). qPCR further confirmed that the expression of the M1 markers iNOS and CCL2 was increased by LPS. After treatment with Nrf2-ADSCs, the expression of M1 markers decreased, but the downregulation of Nrf2 expression in ADSCs reversed the inhibitory effects of ADSCs against LPS-induced M1 microglial phenotype polarization. Nrf2ADSC treatment also promoted the expression of the $M 2$ markers Ym1 and Arg1 (Fig. 6h-k). Taken together, these results suggested that ADSCs reversed LPS-induced nerve cell injury and inflammatory cytokine expression by suppressing $M 1$ polarization and TLR4/NFKB activation via the Nrf2/HO-1 signal pathway.

\section{DISCUSSION}

Depression has been on the rise in recent years as a result of increases in stress in everyday life. Increasing evidence has indicated that the excessive secretion of proinflammatory cytokines, including MCP-1, IL- $1 \beta, \mathrm{IL}-6$, and TNF- $\alpha$, results in behavior similar to that of depression [28]. Here, we also found that MCP-1, IL-1 $\beta, I L-6$, and TNF- $\alpha$ expression was increased after the induction of depression in both in vivo and in vitro experiments. Interestingly, ADSC treatment suppressed MCP-1, IL-1 $\beta, I L-6$, and TNF-a expression. The results also showed that ADSC treatment improved depressive-like behaviors in the SPT, TST, and FST, which is in agreement with previous reports regarding depression and inflammation [36]. We also found that ADSC treatment increased the expression levels of BDNF and TrkB, which were decreased after depression. The activation of the BDNF-TrkB signaling pathway was previously shown to regulate brain inflammation and protect against hippocampal injury $[37,38]$, indicating that the protective effect elicited by ADSCs may be related to reduced signs of depression.

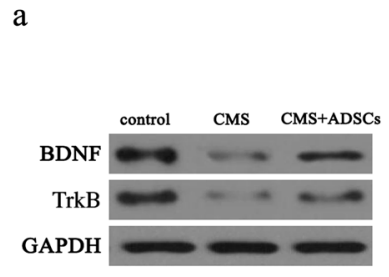

d

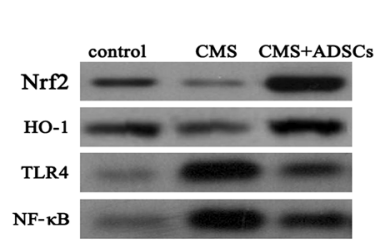

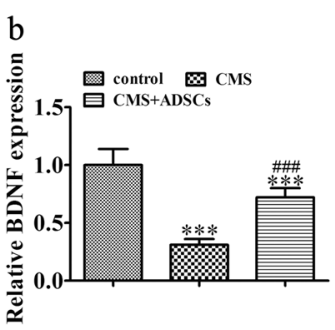

$\mathrm{e}$
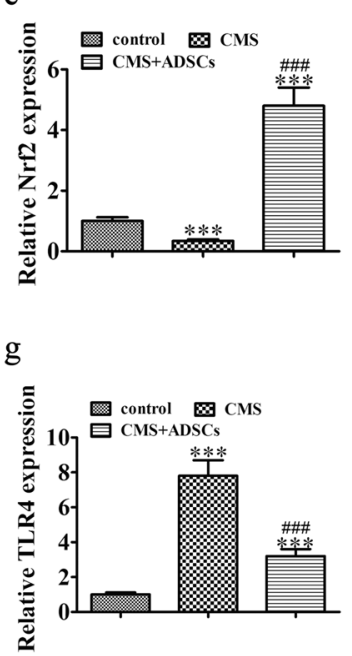
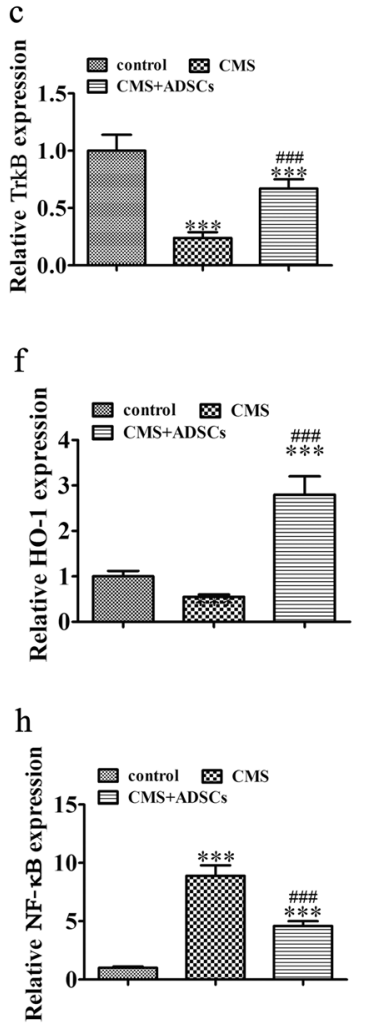

Fig. 4 Nrf2 and TLR4 played roles in the ADSC-mediated protective effect on neurons. a-c Western blot analysis showing the expression of BDNF and TrkB. The data are expressed as the mean \pm SEM. ${ }^{* * *} P<0.001$ vs control; ${ }^{\# \# \# ~} P<0.001$ vs CMS. $\mathbf{d}-\mathbf{h}$ Western blot analysis showing the

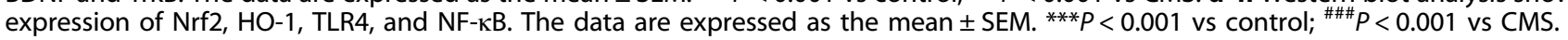




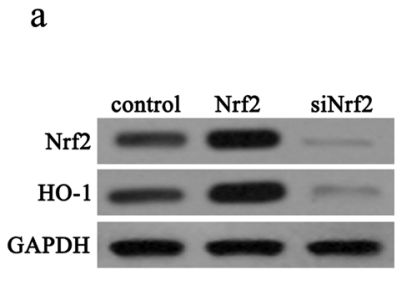

b

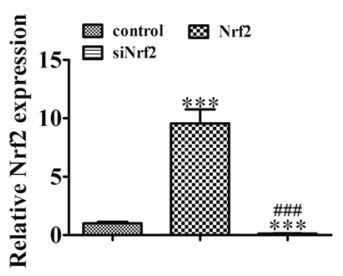

c

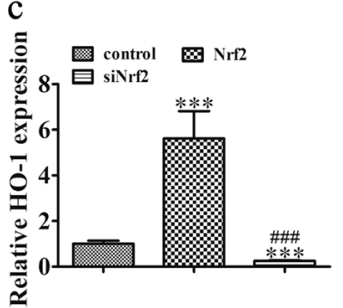

Fig. 5 The expression of Nrf2 and HO-1 in ADSCs after transfection with an Nrf2 overexpression vector or siRNA targeting Nrf2 (siNrf2). a-c Western blot analysis showing the expression of Nrf2 and HO-1 in ADSCs. The data are expressed as the mean \pm SEM. ${ }^{* * *} P<0.001$ vs control; ${ }^{\# \#} P<0.001$ vs Nrf2.

a

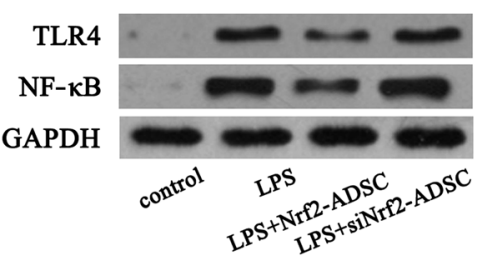

d

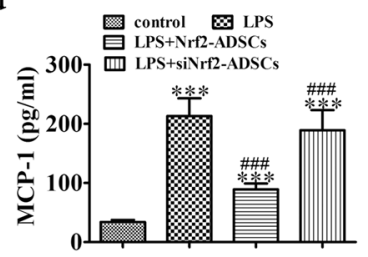

$\mathrm{g}$

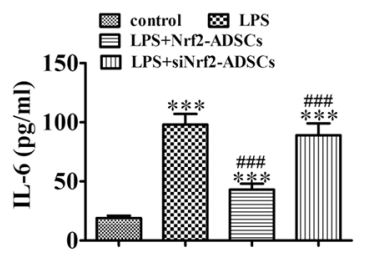

j

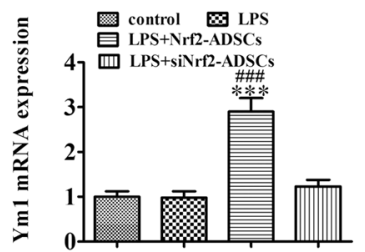

b

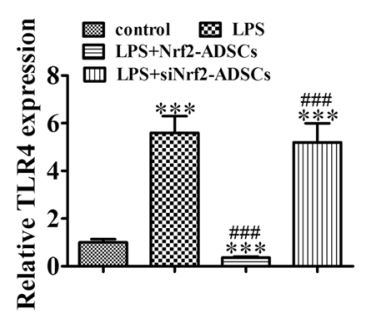

e

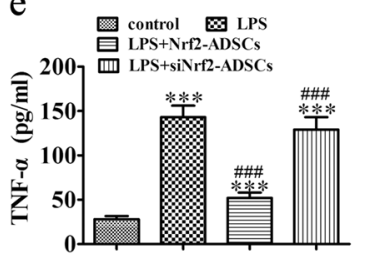

$\mathrm{h}$

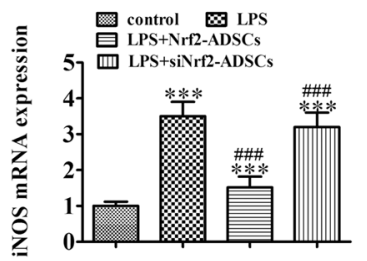

c

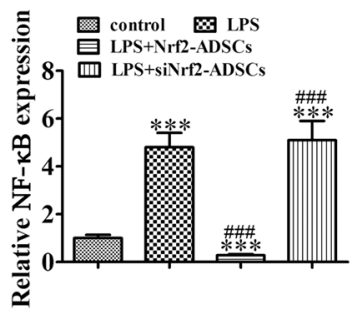

f

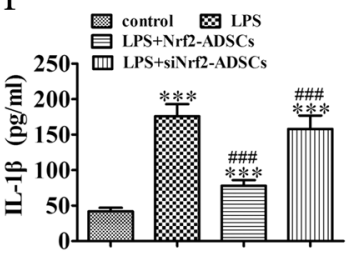

i

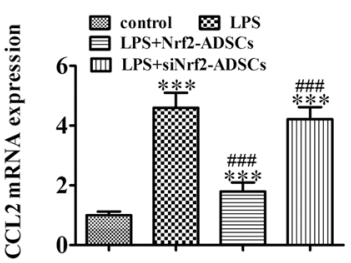

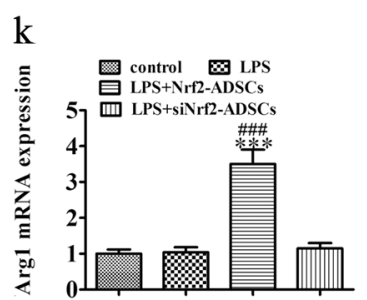

Fig. 6 ADSC and BV2 cell cocultures showed that Nrf2 plays a role in ADSC-mediated protective effects against LPS-induced inflammatory factor expression in BV2 microglial cells. a-c Western blot analysis showing the expression of TLR4 and NF- $\mathrm{B} B$ in BV2 cells. The data are expressed as the mean \pm SEM. ${ }^{* * *} P<0.001$ vs control; ${ }^{\# \# \#} P<0.001$ vs LPS. d-g ELISA analysis showing the expression of the inflammatory factors MCP-1, TNF- $\alpha, \mathrm{IL}-1 \beta, \mathrm{IL}-6$ in the cellular supernatant. The data are expressed as the mean \pm SEM. ${ }^{* * *} P<0.001$ vs control;

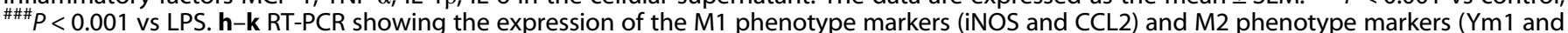
Arg1) in brain tissue. The data are expressed as the mean \pm SEM. ${ }^{* * *} P<0.001$ vs control; ${ }^{\# \#} P<0.001$ vs LPS.

Further investigation found that depression promoted TLR4/ NF-KB activation and concurrently downregulated the Nrf2/HO1 signaling pathway. In contrast, ADSC treatment increased Nrf2/HO-1 signaling and decreased TLR4/NF-KB activation. TLR4 signaling leads to the activation of the JNK signaling cascade to induce neuroinflammation and neurodegeneration and/or interferes with the $\mathrm{Bcl}-2$ family of proteins to activate the mitochondrial apoptotic pathway $[39,40]$. This TLR4 activation triggers the production of TNF- $\alpha$ and IFN- $\gamma$ in macrophages via MyD88 [41]. Specifically, M1 macrophages induce TLR4 activation, which stimulates STAT1/STAT2/IRF9 to bind with interferonstimulated response elements to induce a feedforward loop of LPS-induced gene expression to promote macrophage activation $[42,43]$. In vitro experiments found that silencing Nrf2 in ADSCs 
inhibited the anti-inflammatory effect, which is consistent with previously reported studies [44, 45]. We also found that Nrf2 played an important role in ADSC-mediated microglial phenotype regulation. Specifically, ADSC treatment suppressed the LPSinduced M1 phenotype and promoted the M2 phenotype. These results are in agreement with those of previous studies that showed that Nrf2 activation is related to switching from the M1 to the $M 2$ phenotype $[18,46]$. As the immune cells of the CNS, microglia can influence neurogenesis through the $\mathrm{M} 1$ and M2 subtypes; one study indicated that switching of the microglial phenotype is a possible treatment for depression [47]. Activated receptor-gamma-induced M2 microglia (alternatively activated microglia) also promote the proliferation and differentiation of neural precursor cells [48]. Our study found that Nrf2 activation played an important role in switching M1 phenotype microglia to the $M 2$ phenotype [49].

Meanwhile, a previous study verified that Nrf2 expression inhibits hyperglycemia-induced peripheral nerve injury by suppressing the TLR4/NF-KB signaling pathway [50]. The expression of Nrf2 also suppresses TLR4/NF-kB-mediated inflammatory cytokine expression [51, 52]. Therefore, the present study was undertaken to investigate the mechanism by which ADSCs reduce depressivelike behaviors and the expression of proinflammatory cytokines by Nrf2 regulation.

In summary, our study indicated that ADSCs can protect against CMS-induced depressive-like behaviors by suppressing inflammation, which is accompanied by microglial phenotypic switching towards the M2 phenotype. We believe these results are dependent on Nrf2 expression in ADSCs, which indicates their possible use in depression treatment.

\section{ACKNOWLEDGEMENTS}

This work was supported by grants from the National Natural Science Foundation of China (31771184).

\section{AUTHOR CONTRIBUTIONS}

HW, XW, and JLJ designed the study and wrote the protocols; XH, YW, and GQF managed the literature retrieval and performed the experimental work and data analysis; WJL, JD, and XW wrote the first draft of the manuscript. All authors contributed to and have approved the final manuscript.

\section{ADDITIONAL INFORMATION}

Competing interests: The authors declare no competing interests.

\section{REFERENCES}

1. Wong ML, Licinio J. Research and treatment approaches to depression. Nat Rev Neurosci. 2001;2:343-51.

2. Dantzer R, O'Connor JC, Freund GG, Johnson RW, Kelley KW. From inflammation to sickness and depression: when the immune system subjugates the brain. Nat Rev Neurosci. 2008;9:46-56.

3. Miller $\mathrm{AH}$, Raison $\mathrm{CL}$. The role of inflammation in depression: from evolutionary imperative to modern treatment target. Nat Rev Immunol. 2016;16:22-34.

4. Aguilar-Valles A, Kim J, Jung S, Woodside B, Luheshi GN. Role of brain transmigrating neutrophils in depression-like behavior during systemic infection. Mol Psychiatry. 2014;19:599-606.

5. Yirmiya R, Rimmerman N, Reshef R. Depression as a microglial disease. Trends Neurosci. 2015;38:637-58.

6. Preston TC, Shelton RC. Treatment resistant depression: strategies for primary care. Curr Psychiatry Rep. 2013;15:370.

7. Hanisch UK, Kettenmann H. Microglia: active sensor and versatile effector cells in the normal and pathologic brain. Nat Neurosci. 2007;10:1387-94.

8. Tambuyzer BR, Ponsaerts P, Nouwen EJ. Microglia: gatekeepers of central nervous system immunology. J Leukoc Biol. 2009;85:352-70.

9. Steiner J, Bielau H, Brisch R, Danos P, Ullrich O, Mawrin C, et al. Immunological aspects in the neurobiology of suicide: elevated microglial density in schizophrenia and depression is associated with suicide. J Psychiatr Res. 2008;42:151-7.
10. Park J, Min JS, Kim B, Chae UB, Yun JW, Choi MS, et al. Mitochondrial ROS govern the LPS-induced pro-inflammatory response in microglia cells by regulating MAPK and NF-kappaB pathways. Neurosci Lett. 2015;584:191-6.

11. Zhao Q, Xie X, Fan Y, Zhang J, Jiang W, Wu X, et al. Phenotypic dysregulation of microglial activation in young offspring rats with maternal sleep deprivationinduced cognitive impairment. Sci Rep. 2015:5:9513.

12. Kobayashi K, Imagama S, Ohgomori T, Hirano K, Uchimura K, Sakamoto K, et al. Minocycline selectively inhibits M1 polarization of microglia. Cell Death Dis. 2013;4:e525.

13. Lu Y, Yang YY, Zhou MW, Liu N, Xing HY, Liu XX, et al. Ketogenic diet attenuates oxidative stress and inflammation after spinal cord injury by activating Nrf2 and suppressing the NF-kappaB signaling pathways. Neurosci Lett. 2018;683:13-8.

14. Paraswani N, Thoh M, Bhilwade HN, Ghosh A. Early antioxidant responses via the concerted activation of NF-kappaB and Nrf2 characterize the gamma-radiationinduced adaptive response in quiescent human peripheral blood mononuclear cells. Mutat Res. 2018:831:50-61.

15. Nakano $Y$, Shimazawa M, Ojino K, Izawa H, Takeuchi H, Inoue $Y$, et al. Toll-like receptor 4 inhibitor protects against retinal ganglion cell damage induced by optic nerve crush in mice. J Pharmacol Sci. 2017;133:176-83.

16. Yang MY, Yu QL, Huang YS, Yang G. Neuroprotective effects of andrographolide derivative $\mathrm{CX}-10$ in transient focal ischemia in rat: Involvement of Nrf2/AE and TLR/NF-kappaB signaling. Pharmacol Res. 2019;144:227-34.

17. Feng G, Sun B, Liu HX, Liu QH, Zhao L, Wang TL. EphA2 antagonism alleviates LPS-induced acute lung injury via Nrf2/HO-1, TLR4/MyD88 and RhoA/ROCK pathways. Int Immunopharmacol. 2019;72:176-85.

18. Wang $Y$, Huang $Y, X u Y$, Ruan W, Wang $H$, Zhang $Y$, et al. A dual AMPK/Nrf2 activator reduces brain inflammation after stroke by enhancing microglia M2 polarization. Antioxid Redox Signal. 2018;28:141-63.

19. Kang HS, Choi SH, Kim BS, Choi JY, Park GB, Kwon TG, et al. Advanced properties of urine derived stem cells compared to adipose tissue derived stem cells in terms of cell proliferation, immune modulation and multi differentiation. J Korean Med Sci. 2015:30:1764-76.

20. Guo J, Guo S, Wang Y, Yu Y. Promoting potential of adipose derived stem cells on peripheral nerve regeneration. Mol Med Rep. 2017;16:7297-304.

21. Xie S, Lu F, Han J, Tao K, Wang H, Simental A, et al. Efficient generation of functional Schwann cells from adipose-derived stem cells in defined conditions. Cell Cycle. 2017;16:841-51.

22. Jahromi M, Razavi S, Amirpour N, Khosravizadeh Z. Paroxetine can enhance neurogenesis during neurogenic differentiation of human adipose-derived stem cells. Avicenna J Med Biotechnol. 2016;8:152-8.

23. Wang L, Liu LP, Gu XL, Wang M, Liu LM. Implantation of adipose-derived stem cells cures the optic nerve injury on rats through inhibiting the expression of inflammation factors in the TLR4 signaling pathway. Eur Rev Med Pharmacol Sci. 2018;22:1196-202.

24. Jin $R$, Shen $M, Y u$ L, Wang $X$, Lin X. Adipose-derived stem cells suppress inflammation induced by IL-1beta through down-regulation of P2X7R mediated by miR-373 in chondrocytes of osteoarthritis. Mol Cells. 2017;40: 222-9.

25. Zhang J, Xie X, Tang M, Zhang B, Zhao Q, Han Y, et al. Salvianolic acid B promotes microglial M2-polarization and rescues neurogenesis in stress-exposed mice. Brain Behav Immun. 2017;66:111-24.

26. Steru $L$, Chermat $R$, Thierry $B$, Simon $P$. The tail suspension test: a new method for screening antidepressants in mice. Psychopharmacology (Berl). 1985;85:367-70.

27. Porsolt RD, Bertin A, Jalfre M. Behavioral despair in mice: a primary screening test for antidepressants. Arch Int Pharmacodyn Ther. 1977;229:327-36.

28. Jia KK, Ding H, Yu HW, Dong TJ, Pan Y, Kong LD. Huanglian-Wendan decoction inhibits NF-kappaB/NLRP3 inflammasome activation in liver and brain of rats exposed to chronic unpredictable mild stress. Mediators Inflamm. 2018;2018:3093516.

29. Zhou F, Gao S, Wang L, Sun C, Chen L, Yuan P, et al. Human adipose-derived stem cells partially rescue the stroke syndromes by promoting spatial learning and memory in mouse middle cerebral artery occlusion model. Stem Cell Res Ther. 2015;6:92.

30. Yang YC, Liu BS, Shen CC, Lin CH, Chiao MT, Cheng HC. Transplantation of adipose tissue-derived stem cells for treatment of focal cerebral ischemia. Curr Neurovasc Res. 2011;8:1-13.

31. Mattei D, Djodari-Irani A, Hadar R, Pelz A, de Cossio LF, Goetz T, et al. Minocycline rescues decrease in neurogenesis, increase in microglia cytokines and deficits in sensorimotor gating in an animal model of schizophrenia. Brain Behav Immun. 2014;38:175-84

32. Lu L, Li X, Xu P, Zheng $Y$, Wang $X$. Tenuigenin down-regulates the release of nitric oxide, matrix metalloproteinase- 9 and cytokines from lipopolysaccharidestimulated microglia. Neurosci Lett. 2017;650:82-8. 
33. Zhao H, Alam A, Chen Q, A Eusman M, Pal A, Eguchi S, et al. The role of microglia in the pathobiology of neuropathic pain development: what do we know? $\mathrm{Br} J$ Anaesth. 2017;118:504-16.

34. Chong PN, Sangu M, Huat TJ, Reza F, Begum T, Yusoff AAM, et al. Trkb-IP3 pathway mediating neuroprotection in rat hippocampal neuronal cell culture following induction of kainic acid. Malays J Med Sci. 2018;25:28-45.

35. Sun ZQ, Meng FH, Tu LX, Sun L. Myricetin attenuates the severity of seizures and neuroapoptosis in pentylenetetrazole kindled mice by regulating the BDNF-TrkB signaling pathway and modulating matrix metalloproteinase-9 and GABAA. Exp Ther Med. 2019;17:3083-91.

36. Kohler O, Krogh J, Mors O, Benros ME. Inflammation in depression and the potential for anti-inflammatory treatment. Curr Neuropharmacol. 2016; 14:732-42.

37. Jin $M$, Sheng W, Han L, He Q, Ji X, Liu K. Activation of BDNF-TrkB signaling pathway-regulated brain inflammation in pentylenetetrazole-induced seizures in zebrafish. Fish Shellfish Immunol. 2018;83:26-36.

38. Gao J, Xiong B, Zhang B, Li S, Huang N, Zhan G, et al. Sulforaphane alleviates lipopolysaccharide-induced spatial learning and memory dysfunction in mice: the role of BDNF-mTOR signaling pathway. Neuroscience. 2018;388:357-66.

39. Putcha GV, Le S, Frank S, Besirli CG, Clark K, Chu B, et al. JNK-mediated BIM phosphorylation potentiates BAX-dependent apoptosis. Neuron. 2003;38: 899-914.

40. Tournier C, Hess P, Yang DD, Xu J, Turner TK, Nimnual A, et al. Requirement of JNK for stress-induced activation of the cytochrome c-mediated death pathway. Science. 2000;288:870-4.

41. Vieira TDS, Rugani JN, Nogueira PM, Torrecilhas AC, Gontijo CMF, Descoteaux A, et al. Intraspecies polymorphisms in the lipophosphoglycan of L. braziliensis differentially modulate macrophage activation via TLR4. Front Cell Infect Microbiol. 2019;9:240.

42. Yamaguchi R, Sakamoto A, Yamamoto $T$, Narahara S, Sugiuchi $H$, Yamaguchi $Y$. Differential regulation of IL-23 production in M1 macrophages by TIR8/SIGIRR through TLR4- or TLR7/8-mediated signaling. Cytokine. 2017;99:310-5.
43. Toshchakov V, Jones BW, Perera PY, Thomas K, Cody MJ, Zhang S, et al. TLR4, but not TLR2, mediates IFN-beta-induced STAT1alpha/beta-dependent gene expression in macrophages. Nat Immunol. 2002;3:392-8.

44. Yang X, Chen S, Shao Z, Li Y, Wu H, Li X, et al. Apolipoprotein E deficiency exacerbates spinal cord injury in mice: inflammatory response and oxidative stress mediated by NF-kappaB signaling pathway. Front Cell Neurosci. 2018;12:142.

45. Wen Z, Hou W, Wu W, Zhao Y, Dong X, Bai X, et al. 6'-O-galloylpaeoniflorin attenuates cerebral ischemia reperfusion-induced neuroinflammation and oxidative stress via PI3K/Akt/Nrf2 activation. Oxid Med Cell Longev. 2018;2018:8678267.

46. MacDowell KS, Caso JR, Martin-Hernandez D, Moreno BM, Madrigal JLM, Mico JA, et al. The atypical antipsychotic paliperidone regulates endogenous antioxidant/ anti-inflammatory pathways in rat models of acute and chronic restraint stress. Neurotherapeutics. 2016;13:833-43.

47. Zhang L, Zhang J, You Z. Switching of the microglial activation phenotype is a possible treatment for depression disorder. Front Cell Neurosci. 2018;12:306.

48. Zhao Q, Wang Q, Wang J, Tang M, Huang S, Peng K, et al. Maternal immune activation-induced PPARgamma-dependent dysfunction of microglia associated with neurogenic impairment and aberrant postnatal behaviors in offspring. Neurobiol Dis. 2019;125:1-13.

49. Michel HE, Menze ET. Tetramethylpyrazine guards against cisplatin-induced nephrotoxicity in rats through inhibiting HMGB1/TLR4/NF-kappaB and activating Nrf2 and PPAR-gamma signaling pathways. Eur J Pharmacol. 2019;857:172422.

50. Tang W, Chen X, Liu H, Lv Q, Zou J, Shi Y, et al. Expression of Nrf2 promotes schwann cell-mediated sciatic nerve recovery in diabetic peripheral neuropathy. Cell Physiol Biochem. 2018;46:1879-94.

51. Yan J, Li J, Zhang L, Sun Y, Jiang J, Huang Y, et al. Nrf2 protects against acute lung injury and inflammation by modulating TLR4 and Akt signaling. Free Radic Biol Med. 2018;121:78-85.

52. Hsieh YH, Deng JS, Chang YS, Huang GJ. Ginsenoside Rh2 ameliorates lipopolysaccharide-induced acute lung injury by regulating the TLR4/PI3K/Akt/ mTOR, Raf-1/MEK/ERK, and keap1/Nrf2/HO-1 signaling pathways in mice. Nutrients. 2018;10:1208-17. 